\title{
Erratum: Model-based projections of Zika virus infections in childbearing women in
} the Americas

T. Alex Perkins, Amir S. Siraj, Corrine W. Ruktanonchai, Moritz U. G. Kraemer and Andrew J. Tatem

Nature Microbiology 1, 16126 (2016); published online 25 July 2016; corrected 20 March 2017

In the original version of this Letter, the name of author T. Alex Perkins was coded wrongly, resulting in it being incorrect when exported to citation databases. This has now been corrected, though no visible changes will be apparent. 